ARTÍCULOS 



\title{
ANÁLISIS ESPACIO-TEMPORAL DE LAS LLUVIAS TORRENCIALES EN LA CIUDAD DE MÁLAGA
}

\author{
José María Senciales González \\ José Damián Ruiz Sinoga \\ Departamento de Geografía. Universidad de Málaga \\ senciales@uma.es, sinoga@uma.es
}

\section{RESUMEN}

Se determina la distribución espacial de la probabilidad de lluvias torrenciales en Málaga capital, donde hay antecedentes de graves inundaciones $(1907,1956,1978,1979,1989 \ldots)$, y se profundiza en las variables que inciden en esta torrencialidad (altitud, exposición local y del relieve, tipo de tiempo, recurrencia), analizando su tendencia en contraste con los escenarios de cambio climático. Los resultados muestran como la distribución de la frecuencia de los aguaceros torrenciales es muy superior a la que los análisis de un solo observatorio llegan a mostrar mediante análisis probabilístico.

Palabras clave: Torrencialidad, tipos de tiempo, periodos de retorno, incidencia del relieve, escenarios de cambio climático.

\begin{abstract}
The objective of this paper is to show a map of heavy rains probabilities in Malaga city, where there are several records of serious floods $(1907,1956,1978,1979,1989 \ldots)$. We explore parameters that influence heavy rains (altitude, local winds exposure, weather type, recurrence...) and we analyze trends of these events in contrast to global climate change settings. The results show how the distribution of the frequency of torrential downpours is far superior to the analysis of a single Observatory arriving to show through probabilistic analysis.
\end{abstract}

Fecha de recepción: diciembre 2010.

Fecha de aceptación: diciembre 2012. 
Key words: Heavy rain, Weather type, recurrence periods, relief effects, global climate change settings.

\section{INTRODUCCIÓN, ANTECEDENTES Y OBJETIVOS}

La variabilidad espacial y temporal de las precipitaciones de clima mediterráneo ha sido analizada en numerosos estudios (Pons y Soriano, 1994; De Luis et al., 1997; Pascual et al., 2001; Martin Vide, 2004; Llasat et al., 2005; Neppel et al., 2007; Rodrigo y Barriendos, 2008; Lana et al., 2009; Turco y Llasat, 2011), así como los episodios de la dinámica atmosférica que suelen intervenir en tales eventos (Llasat et al., op. cit.; Martín et al., 2006; Martin Vide et al., 2008; Camarasa et al., 2010). Sin embargo, aun considerando la gran variabilidad espacial que estos mismos estudios reconocen, algunas áreas del Mediterráneo apenas se han estudiado en profundidad, a pesar de que existen datos más que suficientes. Es el caso de la ciudad de Málaga, donde se cuenta con datos pluviométricos continuos y diarios desde 1935 (y totalizados desde 1878).

La comparación sistemática de situaciones sinópticas con eventos torrenciales permite conocer cuáles son los tipos de tiempo que propician situaciones de riesgo meteorológico en localizaciones concretas, dado que es obvio que la topografía introduce importantes modificaciones en el comportamiento de las masas de aire, en especial en áreas como la vertiente mediterránea, donde los relieves superan con bastante frecuencia los mil metros de altura en cortos recorridos.

Son numerosos los trabajos que han atendido a la distribución de las particularidades mesoclimáticas del clima mediterráneo. Son clásicos los estudios de Elías y Ruiz (1979), Font Tullot (1983), López Gómez (1969, 1983), Albentosa (1991) o Capel Molina (2000) sobre el clima general de la península ibérica, referenciando algunas particularidades del mismo en determinados lugares del mediterráneo, en especial aquellos en los que la torrencialidad catastrófica suele ser más recurrente, como es el Levante, llegando a hablarse de «supuesto monzón de la Península Ibérica» (López Gómez, 1969). Los Sistemas Convectivos de Mesoscala (Zipser, 1982) dependen de flujos distintos en el área de Málaga.

Como señala Capel (1990), «...cada vez que ocurre un desastre de origen meteorológico, lo más que se hace es un estudio a posteriori de los parámetros que confluyeron para producir unos hidrometeoros fuera de lo común». Aunque el progreso de las técnicas de análisis meteorológico gracias a la existencia de ordenadores cada día más potentes conduzcan a alejar esta idea mediante previsiones cada vez más fiables, no deja de ser cierto que la localización precisa de los efectos que se prevén sigue estando lejos de nuestro alcance. A escala microclimática la previsión, hoy por hoy, es impensable. En una comarca podemos estar alertados ante lluvias torrenciales en su ámbito, prevenir problemas de tráfico, alertar de avenidas fluviales o de inundaciones. Sin embargo, la prevención en una gran ciudad es mucho más difícil de abordar, siendo difícil conseguir poco más que una limpieza adecuada del alcantarillado o de los cauces fluviales que la surquen.

Son escasos pero útiles los estudios concretos realizados sobre la ciudad de Málaga. Elias y Ruiz (1979), en su análisis sobre precipitaciones máximas en España, abordan varias estaciones de la ciudad, aunque se trate de un trabajo antiguo. Senciales (1995 y 1997), aborda la intensi- 
dad de las precipitaciones en Málaga o en su entorno. También es imprescindible el trabajo de Capel (1990), que analiza un episodio de especial intensidad en la historia reciente de la ciudad.

En el presente estudio se aborda y sistematiza el análisis de las precipitaciones torrenciales históricas de la ciudad de Málaga y hayan sido documentadas en registros pluviométricos, con objeto de cartografiar la probabilidad de suceso de eventos superiores a $100 \mathrm{~mm}$ ante determinadas condiciones atmosféricas.

\section{FUENTES Y MÉTODOS}

\subsection{Localización}

Málaga se localiza en un área intermedia de incidencia de los procesos de origen atlántico y de aquéllos que se originan en el Mediterráneo, lo que determina que no cumpla en ningún caso con las características clásicas de ninguna de las dos áreas. Por esta razón, tampoco pueden generalizarse en Málaga los eventos que afectan a dichas áreas; no sólo no afectan de igual modo, sino que además es frecuente que numerosos eventos de intensidad extrema acaecidos en Málaga pasen desapercibidos en el Estrecho o en Levante, por citar áreas cercanas; y viceversa.

El relieve de la ciudad de Málaga posee la peculiaridad de ubicarse al pie de un sistema montuoso que alcanza 500 m. en tan sólo 3 km. (Monte San Antón), o 1.000 m. en 10 km.

Figura 1

LOCALIZACIÓN DEL ÁREA DE ESTUDIO

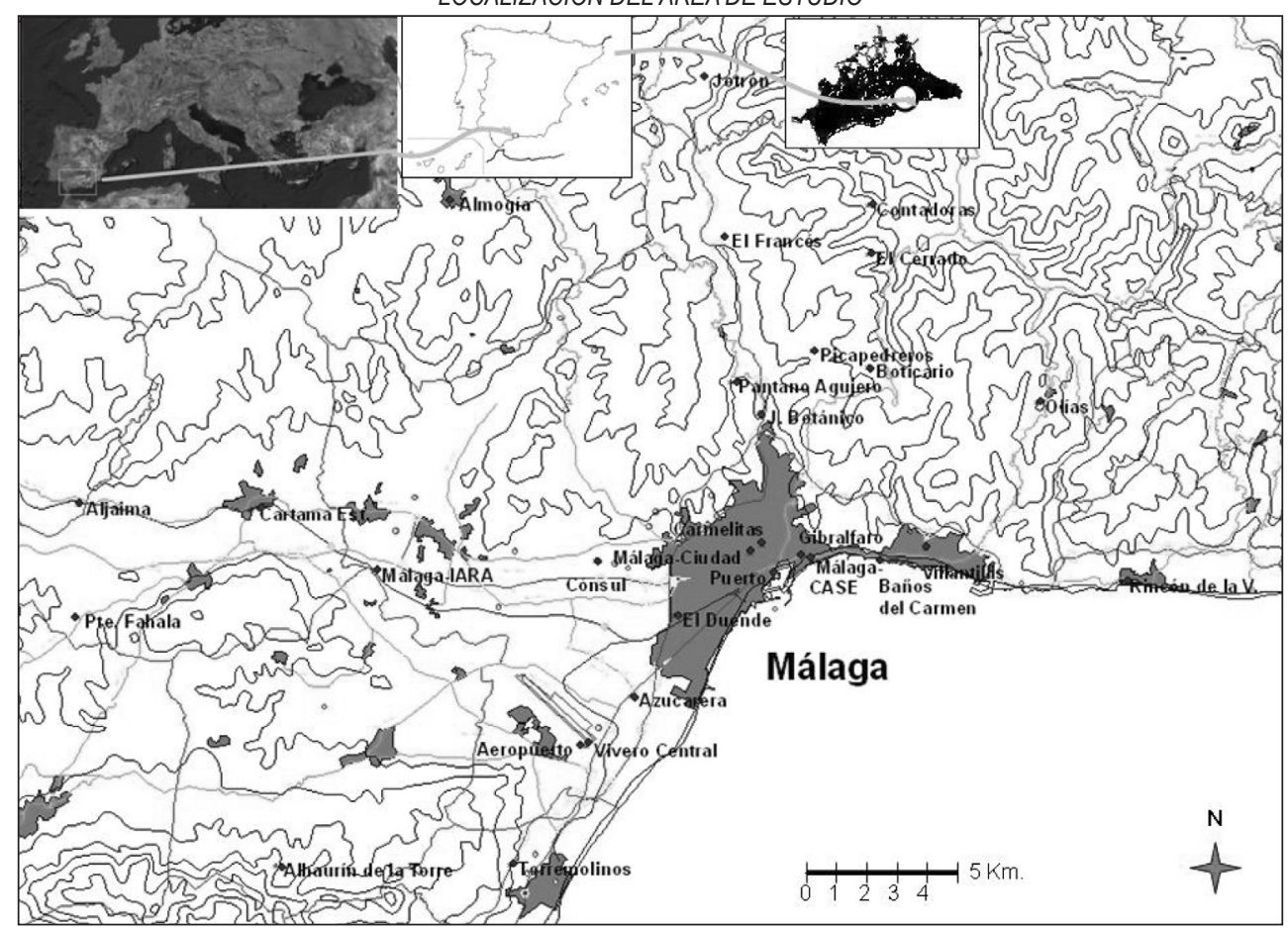


(La Reina). Sin embargo, al oeste se extiende una amplia vega (bajo valle del río Guadalhorce) situada a sotavento de relieves como la serranía de Ronda, próxima a los $2.000 \mathrm{~m}$. de altitud.; o como las sierras de Mijas, Alpujata y Blanca, que superan todas ellas los 1.000. Ello determina un leve efecto föhn sin la entidad de Almería o Murcia, pero que se evidencia en un descenso de las precipitaciones hasta valores inferiores a los $500 \mathrm{~mm} /$ año en el fondo del valle (413,6 en Aljaima) y cercanos a 500 en diversos sectores de Málaga ciudad: Atabal (458 mm), Limonero (481 mm), Comisaría de aguas (483 mm)... que, no obstante, se elevan en el aeropuerto de Málaga (538 mm) y en la zona de los Montes: Olías (553 mm), Boticario (576 mm), Contadoras (643 mm), por citar algunas.

\subsection{Métodos}

Se ha restringido el estudio a los eventos torrenciales, entendiendo por tales aquéllos que superen 100 mm en 24 horas. El mapa de precipitación máxima en 24 horas elaborado por Elías y Ruiz (1979) asignaba a la ciudad de Málaga un valor máximo de entre 220 y 240 mm para un periodo de retorno de 100 años; y un valor máximo de $60 \mathrm{~mm} / \mathrm{h}$. para un periodo de retorno de 10 años. Las estimaciones probabilísticas para la ciudad de Málaga (estación Málaga-CASE) realizadas por Senciales (1997) señalan una recurrencia de entre 10 y 25 años para tales eventos; pero al tratar un mayor número de estaciones, esta recurrencia parece ser mucho más corta, por lo que se aborda el estudio de todas las estaciones periféricas e internas a la ciudad con objeto de realizar una estimación múltiple y no basada en un único punto que pueda estar condicionado por su localización.

Con estas premisas puede catalogarse una serie corta de eventos, a la vez que se trabaja con registros pluviográficos y pluviométricos, éstos últimos más extensos en el tiempo y en el espacio.

A partir del criterio ausencia-presencia del registro de un determinado evento en cualquier punto de la ciudad, se han tomado datos de 29 estaciones tanto internas como periféricas a la ciudad de Málaga (Figura 1) que abarcan desde 1950 hasta 2009. Así, en el mejor de los casos se ha dispuesto de datos de 15 estaciones para un mismo evento, siendo lo habitual contar con datos de entre 10 y 12 de ellas desde 1955. En cada una se ha referenciado la fecha y volumen de los eventos torrenciales, así como la cantidad registrada en momentos en los que en cualquier otra estación se haya producido este hecho, lo que nos lleva a depurar la tabla y realizar una nueva registrando sólo los eventos superiores a $100 \mathrm{~mm} / 24 \mathrm{~h}$. Para lograr una identificación con los sectores de la ciudad se han agrupado en función de su posición fisiográfica: 1) Costa occidental-Valle del Guadalhorce (9 estaciones: a-i); 2) Sector urbano occidental (5 estaciones: j-n); 3) Sector urbano oriental-Rincón de la Victoria (5 estaciones: ñ-r); Montes de Málaga (10 estaciones: o- $\beta$ ). Por último, se ha añadido la dirección del viento predominante durante el evento: $1=\mathrm{N}, 2=\mathrm{NE}, 3=\mathrm{E}, 4=\mathrm{SE}, 5=\mathrm{S}, 6=\mathrm{SO}, 7=\mathrm{O}, 8=\mathrm{NO}$; como puede verse, las direcciones de componente norte (N, NE y NO) están ausentes en los eventos seleccionados.

A partir de los archivos históricos de la aplicación de Wetterzentrale (2010), con datos de superficie y $500 \mathrm{hPa}$., entre otros, se ha podido realizar una interpretación y catalogación de la situación sinóptica que determinó la lluvia torrencial, siguiendo el modelo de Capel (2000), por una parte, y las definiciones de Martín León (2003) sobre fenómenos de mesoescala. 
Se ha atendido al número de casos registrado en cada zona a lo largo de la serie, valorando el fenómeno en cada zona en conjunto. Así mismo, se ha realizado una cartografía de la probabilidad de recurrencia de las precipitaciones aplicando el modelo probabilístico de Poisson ya empleado por Senciales; estos resultados se han introducido en el programa ArcMap 9.3.1 para obtener un mapa de distribución a partir de las coordenadas de cada valor empleando la aplicación Inverse Distance Weighted (IDW) de Spatial Analyst.

\section{RESULTADOS}

\subsection{Tipología de los eventos torrenciales}

Se han registrado 30 eventos torrenciales en la ciudad de Málaga entre 1950 y 2008. Durante este periodo dicho tipo de eventos están ausentes en los meses de abril a agosto (Tabla 1) en todos los registros, produciéndose la máxima frecuencia de lluvias extremas en el mes de noviembre, seguido del mes de febrero.

Tabla 1

PORCENTAJE DE EVENTOS > 100 MM/24 H POR MES

\begin{tabular}{|l|c|c|c|c|c|c|c|}
\hline Mes & S & O & N & D & E & F & M \\
\hline \% casos & 16.66 & 10.00 & 26.66 & 10.00 & 6.66 & 20.00 & 10.00 \\
\hline
\end{tabular}

El tipo de tiempo que suele provocar estos eventos (Tabla 2) se asocia a situaciones de levante (en superficie) con diferente componente $(E+S E+S)$, ya que el tipo de tiempo que predomina es el suroeste, el mismo que frecuentemente genera precipitaciones ordinarias en Málaga (Senciales, 1995). Ambos se asocian con frecuencia a perturbaciones africanas reactivadas y cargadas de humedad en el Mediterráneo, en tanto que el sur, aunque puede estar asociado a perturbaciones de origen netamente atlántico (del golfo de Cádiz), afecta a Málaga después atravesar el norte de África y el Mediterráneo, por lo que presenta un comportamiento semejante al levante.

Tabla 2

PORCENTAJE DE EVENTOS > 100 MM/24 H SEGÚN TIPO DE TIEMPO

\begin{tabular}{|l|c|c|c|c|c|}
\hline Tipo de tiempo & O & SO & S & SE & E \\
\hline \% casos & 3.33 & 40.00 & 10.00 & 23.33 & 23.33 \\
\hline
\end{tabular}

A ello hay que añadir otros fenómenos combinados con el tipo de tiempo, como son la asociación con sistemas frontales de gran actividad, sistemas frontales ocluidos, flujo de aire frío en altura (descenso latitudinal del chorro polar) y existencia de gota fría típica (ver Tablas 3 y 4) o DANA (Depresión Aislada de Niveles Altos) (Martín León, 2003).

Tabla 3

SITUACIÓN EN ALTURA DURANTE EVENTOS > $100 \mathrm{MM} / 24 \mathrm{H}$

\begin{tabular}{|l|c|c|}
\hline Situación a 500 HPa & Flujo frío & DANA \\
\hline$\%$ casos & 40 & 60 \\
\hline
\end{tabular}


Del total de 30 casos analizados, predomina ligeramente la presencia de DANAs en altura, si bien no deja de ser importante la presencia de flujo frío no aislado. Hay que precisar que en numerosas ocasiones, como suele suceder con los fenómenos de mesoescala, la fuente gráfica consultada no señala la presencia de gota fría aislada del flujo frío de $500 \mathrm{HPa}$, o, si lo hace, está demasiado lejos para afectar a la ciudad de Málaga en el día señalado; sin embargo, no debemos descartar la más que probable presencia de pequeños embolsamientos fríos en niveles ligeramente superiores o inferiores dentro de dicha masa; visto de este modo, todo flujo frío podría entrañar, en rigor, una DANA.

Al combinarlo con la situación en superficie (Tabla 5) destaca el hecho de que la situación más frecuente es la presencia de eventos torrenciales relacionados simplemente con flujo frío en altura y la llegada de un sistema frontal, normalmente de procedencia atlántica. Sin embargo, al combinarlo con el viento predominante destaca la DANA asociada a levante (E y SE) y secundariamente el flujo frío con poniente.

Tabla 4

SITUACIÓN COMBINADA DURANTE EVENTOS > 100 MM/24 H

\begin{tabular}{|c|c|c|c|c|c|}
\hline $\begin{array}{c}\text { Situación } \\
\text { combinada }\end{array}$ & $\begin{array}{c}\text { Flujo frío }+ \\
\text { Frente }\end{array}$ & $\begin{array}{c}\text { Flujo frío }+ \\
\text { Frente ocluido }\end{array}$ & $\begin{array}{c}\text { DANA + } \\
\text { Frente }\end{array}$ & $\begin{array}{c}\text { DANA + } \\
\text { Frente ocluido }\end{array}$ & $\begin{array}{c}\text { DANA + } \\
\text { Depresión }\end{array}$ \\
\hline$\%$ casos & 30 & 10 & 13.33 & 23.33 & 23.33 \\
\hline
\end{tabular}

Tabla 5

SITUACIÓN EN ALTURA Y TIPO DE TIEMPO EN EVENTOS DE > 100 MM/24 H

\begin{tabular}{|l|c|c|c|c|c|}
\hline $\begin{array}{c}\text { Tipo de tiempo } \\
\text { Situación en superficie }\end{array}$ & o & SO & S & SE & E \\
\hline Flujo Frío & 3.33 & 23.33 & 6.67 & 3.33 & 3.33 \\
\hline DANA & 0 & 16.67 & 3.33 & 20.00 & 20.00 \\
\hline
\end{tabular}

(Datos en porcentajes. Elaboración propia)

Así pues, existe una mayor frecuencia de frentes activos (ocluidos o no) con lluvias violentas ligadas a flujo frío que a DANAs, aunque también es destacable la presencia de DANAs ligadas a depresiones, o a frentes ocluidos, efectos ambos comunes en las lluvias torrenciales del levante peninsular. En el caso señalado de DANA + Depresión, es denominada por Martín León (2003) como «borrasca fría». Este hecho se corrobora al contrastar el tipo de tiempo en superficie con el fenómeno desencadenante en superficie: frente, frente ocluido o depresión aislada (tabla 6), que en este último caso está muy ligado al viento de levante en superficie.

Tabla 6

SITUACIÓN EN SUPERFICIE Y TIPO DE TIEMPO EN EVENTOS DE > 100 MM/24 H

\begin{tabular}{|l|c|c|c|c|c|}
\hline $\begin{array}{c}\text { Tipo de tiempo } \\
\text { Situación en superficie }\end{array}$ & O & SO & S & SE & E \\
\hline Frente & 3.33 & 30 & 6.67 & 0 & 3.33 \\
\hline Frente Ocluido & 0 & 6.67 & 3.33 & 16.67 & 6.67 \\
\hline Depresión & 0 & 3.33 & 0 & 6.67 & 13.33 \\
\hline
\end{tabular}

(Datos en porcentajes. Elaboración propia) 
Tabla 7

INTENSIDAD Y DISTRIBUCIÓN DE AGUACEROS >100 MM/24 HEN MÁLAGA

\begin{tabular}{|c|c|c|c|c|c|c|c|c|c|c|}
\hline \multirow{3}{*}{ Fecha aguacero } & \multicolumn{8}{|c|}{$\mathrm{N}^{0}$ de estaciones que registran el evento } & \multicolumn{2}{|c|}{ Eventos $>100 \mathrm{~mm}$} \\
\hline & \multicolumn{2}{|c|}{ Guadalhorce } & \multicolumn{2}{|c|}{ Zona Oeste } & \multicolumn{2}{|c|}{ Zona Este } & \multicolumn{2}{|c|}{ Montes } & & \\
\hline & $\begin{array}{l}>100 \\
\mathrm{~mm}\end{array}$ & $\begin{array}{l}<100 \\
\mathrm{~mm}\end{array}$ & $\begin{array}{c}>100 \\
\mathrm{~mm}\end{array}$ & $\begin{array}{c}<100 \\
\mathrm{~mm}\end{array}$ & $\begin{array}{l}>100 \\
\mathrm{~mm}\end{array}$ & $\begin{array}{l}<100 \\
\mathrm{~mm}\end{array}$ & $\begin{array}{l}>100 \\
\mathrm{~mm}\end{array}$ & $\begin{array}{l}<100 \\
\mathrm{~mm}\end{array}$ & $\begin{array}{l}\text { Event } \\
\text { acum. }\end{array}$ & $\begin{array}{c}\text { Vol. máx. } \\
\text { (mm) }\end{array}$ \\
\hline $28 / 09 / 1950$ & 0 & 1 & 0 & 1 & 0 & 2 & 1 & 3 & 1 & 109 \\
\hline 29/10/1955* & 2 & 1 & 1 & 0 & 2 & 0 & 7 & 0 & 2 & 250.5 \\
\hline 16/11/1956* & 2 & 0 & 1 & 0 & 1 & 0 & 3 & 2 & 3 & 185 \\
\hline 27/09/1957 & 2 & 0 & 1 & 0 & 2 & 0 & 0 & 5 & 4 & 313 \\
\hline $01 / 01 / 1959$ & 1 & 2 & 0 & 1 & 0 & 2 & 0 & 5 & 5 & 144 \\
\hline 18/02/1969 & 1 & 0 & 1 & 0 & 0 & 2 & 2 & 4 & 6 & 112.3 \\
\hline $22 / 02 / 1969$ & 1 & 2 & 1 & 0 & 0 & 2 & 0 & 5 & 7 & 151 \\
\hline 01/09/1969 & 1 & 2 & 0 & 1 & 0 & 2 & 4 & 2 & 8 & 185 \\
\hline 02/11/1972 & 1 & 3 & 0 & 0 & 1 & 2 & 0 & 6 & 9 & 109.9 \\
\hline 29/12/1977 & 0 & 2 & 0 & 1 & 1 & 2 & 0 & 5 & 10 & 169 \\
\hline 10/11/1978* & 3 & 1 & 1 & 0 & 2 & 1 & 2 & 0 & 11 & 240.5 \\
\hline 18/01/1979 & 2 & 3 & 0 & 1 & 0 & 3 & 0 & 3 & 12 & 121.5 \\
\hline 06/11/1982* & 5 & 0 & 1 & 0 & 2 & 1 & 6 & 0 & 13 & 240 \\
\hline $27 / 02 / 1984$ & 2 & 3 & 0 & 1 & 1 & 2 & 0 & 5 & 14 & 208 \\
\hline 18/10/1988 & 3 & 2 & 0 & 2 & 0 & 2 & 1 & 4 & 15 & 144 \\
\hline $14 / 11 / 1989$ & 5 & 0 & 0 & 1 & 0 & 2 & 1 & 4 & 16 & 230 \\
\hline 26/11/1989 & 0 & 1 & 0 & 1 & 0 & 2 & 4 & 0 & 17 & 162 \\
\hline $09 / 12 / 1989$ & 1 & 1 & 0 & 1 & 0 & 3 & 1 & 4 & 18 & 116 \\
\hline 06/03/1991 & 0 & 2 & 0 & 1 & 0 & 2 & 2 & 3 & 19 & 121.5 \\
\hline $15 / 02 / 1994$ & 0 & 2 & 1 & 1 & 0 & 3 & 1 & 5 & 20 & 110 \\
\hline 27/09/1997 & 1 & 2 & 0 & 2 & 0 & 3 & 1 & 4 & 21 & 124.2 \\
\hline 03/02/1998 & 1 & 2 & 0 & 2 & 0 & 1 & 0 & 6 & 22 & 110.7 \\
\hline $26 / 12 / 2000$ & 0 & 1 & 0 & 1 & 0 & 1 & 1 & 4 & 23 & 137 \\
\hline 28/09/2001 & 1 & 1 & 0 & 3 & 1 & 1 & 1 & 5 & 24 & 116.5 \\
\hline $04 / 11 / 2001$ & 0 & 1 & 0 & 3 & 0 & 2 & 1 & 5 & 25 & 110 \\
\hline $24 / 02 / 2004$ & 0 & 2 & 1 & 2 & 0 & 2 & 0 & 6 & 26 & 111.2 \\
\hline $27 / 03 / 2004^{*}$ & 2 & 0 & 2 & 0 & 2 & 0 & 6 & 0 & 27 & 302.4 \\
\hline $07 / 11 / 2006$ & 0 & 2 & 0 & 3 & 1 & 0 & 3 & 1 & 28 & 133 \\
\hline $19 / 03 / 2008$ & 0 & 1 & 0 & 3 & 0 & 2 & 1 & 5 & 29 & 110 \\
\hline $31 / 10 / 2008$ & 2 & 0 & 0 & 3 & 0 & 2 & 1 & 5 & 30 & 135 \\
\hline $\mathrm{N}^{0}$ eventos & 20 & 23 & 10 & 21 & 11 & 25 & 21 & 25 & 30 & 30 \\
\hline$\%$ de eventos & 66.66 & 76.7 & 33.33 & 70.0 & 36.67 & 83.3 & 70.0 & 83.3 & 100 & 100 \\
\hline $\mathrm{N}^{0}$ casos & 39 & 40 & 11 & 35 & 16 & 49 & 50 & 106 & 346 & \\
\hline $\begin{array}{l}\text { Eventos } * 100 \\
\text { / registros }\end{array}$ & 25.3 & 29.1 & 21.7 & 45.6 & 16.9 & 38.5 & 13.5 & 16.0 & 8.7 & \\
\hline $\begin{array}{l}\text { Casos *100/ } \\
\text { Registros }\end{array}$ & 49.4 & 50.6 & 23.9 & 76.1 & 24.6 & 75.4 & 32.1 & 67.9 & 100 & \\
\hline
\end{tabular}

(Elaboración propia. El símbolo * señala aguaceros generalizados. En negrita y cursiva el número de casos y la zona donde se produce el volumen máximo de precipitación en el evento) 
Para atender a la distribución e intensidad de tales eventos se ha analizado la repercusión que tuvieron en cada sector de la ciudad, con objeto de identificar si el factor relieve incidió o no en las características de los mismos. La tabla 7 muestra una realidad común a todos los análisis pluviométricos: la irregularidad en la disponibilidad de datos. Así, de las cinco estaciones disponibles para Málaga Oeste, sólo suele contarse con datos de una a tres estaciones simultáneamente; en Málaga Este, también con cinco, sucede algo parecido; en el sector Guadalhorce, con nueve estaciones, se llega a disponer simultáneamente con datos de hasta un máximo de cinco. Sólo en los Montes de Málaga, donde hay diez estaciones periféricas a la ciudad se llega a contar con hasta siete estaciones simultáneas.

Se han analizado los efectos de cada uno de los 30 aguaceros torrenciales en todas las estaciones disponibles. Así se ha llegado en cada zona a un cómputo total de casos convertido a porcentajes.

Los datos de la tabla 7 revelan que cuando se registra un episodio de alta intensidad, el porcentaje de casos (según número de estaciones de la zona que registra el evento) es superior en la vega del Guadalhorce (en conjunto) al resto del entorno de la ciudad de Málaga, y ello a pesar de que la estación más elevada sólo llega a los 80 m. s.n.m. (Alhaurín de la Torre). Le sigue en importancia la zona de los Montes, con estaciones localizadas en cotas entre 70 y 700 m. A continuación tendríamos la costa oriental urbana, desde Gibralfaro a Rincón de la Victoria, con numerosas colinas abruptas pero de menos de $200 \mathrm{~m}$. de altura. Y finalmente la zona oeste de la ciudad.

\subsection{Análisis multivariante de los efectos de las lluvias torrenciales}

Dadas las características topográficas de la ciudad de Málaga y la localización de las estaciones con que contamos, se probó a agrupar éstas de diverso modo, identificando hasta un máximo de cinco zonas: Guadalhorce, Oeste urbano, Este, Montes Bajos (por debajo de 300 m.) y Montes Altos ( $>300 \mathrm{~m})$. En las primeras pruebas de correlación lineal multivariante con SPSS-18 se obtuvieron las siguientes conclusiones: al analizar los valores máximos por zona, las variables Viento, Altura y Mes muestran resultados poco satisfactorios (en general, salvo datos aislados, por debajo incluso de 0’3); sin embargo, la variable situación de superficie, muestra valores de 0'999, 0'921, 0’828, 0’358 y 0'722 con respecto a cada una de las cinco zonas, en el orden mencionado. Agrupados en cinco zonas, por tanto, es el viento en superficie al considerar los valores medios por zona la variable que más incidencia muestra.

Cuando se analizan tres zonas (Málaga Oeste -fusionando Guadalhorce y Oeste urbano de Málaga-, Málaga Este y Montes -fusionando Montes Altos y Bajos), los resultados son los siguientes: valores de significación inferior a 0’3 al considerar el viento en Superficie, valores de 0'924, 0'909 y 0’341 en la situación en altura; 0'982, 0'871 y 0’424 en la situación de superficie; y 0’245, 0’926 y 0’018 en el mes del año. El análisis de relación multivariante obtiene valores poco significativos (0’478, 0’434 y 0’078), siendo estos los más altos, que se obtienen al relacionar situación en altura y mes del año.

Sin embargo, al analizar tres zonas con los valores máximos de cada zona, se alcanzan nuevamente valores poco significativos en Viento (<0`408), dispares en mes del año (0’92 en Málaga Este y valores de 0'33 en Oeste y 0'033 en Montes) e interesantes en situación en altura y en superficie. En altura se alcanzan valores de 0'968 en Málaga oeste, 0'865 en Este 
y 0'15 en Montes; mientras que en superficie se consiguen valores de 1 en Málaga oeste, 0'828 en Málaga Este y 0'585 en Montes de Málaga. Nuevamente, los resultados multivariantes son poco significativos: 0'443, 0’503 y 0’061 al relacionar Altura y mes del año (en el resto de las variables no se muestran los resultados).

Con cuatro zonas, como se agrupó en un prinicipio y sirvió para la elaboración inicial reflejada en la tabla 7, los resultados son menos significativos. Es decir, la orografía compleja de los Montes de Málaga suele dar lugar a la devaluación de las correlaciones, mientras que la zona de Málaga Oeste suele alcanzar buenas correlaciones con numerosas variables.

\subsection{Recurrencia y tendencia de los eventos torrenciales}

La probabilidad de que se registre en una o más estaciones de la zona un episodio torrencial cuando en cualquier lugar del entorno de Málaga se produce un evento similar (Tabla 7), es mayor en los Montes de Málaga (70\%), seguido de la vega del Guadalhorce, la zona este de la ciudad y, por último, la zona oeste $(33,3 \%)$. No obstante, la probabilidad de que el evento registrado sea inferior a $100 \mathrm{~mm}$ es siempre más elevada, alcanzándose los valores más altos en Montes y zona Este, y los más bajos en Guadalhorce y Zona Oeste.

En 59 años viene a presentarse un promedio de un aguacero torrencial cada 1.97 años en algún punto de la ciudad o entorno de la misma, valor bien distinto de la recurrencia calculada a partir de una única estación. No obstante, aplicando el modelo probabilístico de Poisson:

$$
\mathrm{P}=\mathrm{e}^{-\lambda * \lambda \lambda^{\mathrm{x}} / \mathrm{x} !}
$$

La recurrencia calculada para 30 aguaceros torrenciales en 59 años es de 0'306, o lo que es lo mismo, 1 evento cada 3'27 años en cualquier punto del área estudiada.

De los 30 aguaceros analizados, 20 fueron registrados en más de una estación simultáneamente. Sólo 10 se localizaron en el casco urbano de Málaga, lo que implicaría un periodo de retorno medio de un aguacero torrencial cada 5'9 años en algún punto interno de la ciudad. Según el modelo de Poisson sería 1 cada 7 años.

Sólo 5 de los 30 aguaceros pueden considerarse generalizados, es decir, que han registrado una lluvia torrencial en la mayoría de las estaciones pluviométricas. En este caso sí que coincide la estimación de recurrencia realizada para una única estación. El modelo de Poisson estimaría la recurrencia de aguaceros generalizados en uno cada 12'8 años.

La recurrencia de los eventos torrenciales en conjunto para el entorno de la ciudad de Málaga (Figura 2), muestra una tendencia al incremento más allá de la linealidad que expresaría un gráfico de valores acumulados homogéneos en una serie temporal. Se obtiene una correlación de $\mathrm{R}^{2}=0.9927$ en la ecuación polinómica:

$$
Y=0.0046 x^{2}+0.2055 x+0.9909
$$

Se muestra así una clara tendencia a un incremento progresivo de la frecuencia y número de eventos torrenciales. Los datos en sí son expresivos (ver Tabla 7): entre 1950 y 1979 se registraron en el entorno de Málaga 12 eventos de este tipo; sin embargo, en el mismo periodo de 30 años, desde 1980 a 2009, se registraron 18 casos, es decir, un 50\% más. En ello hay que considerar, además, que el número de estaciones disponibles en ambos periodos 


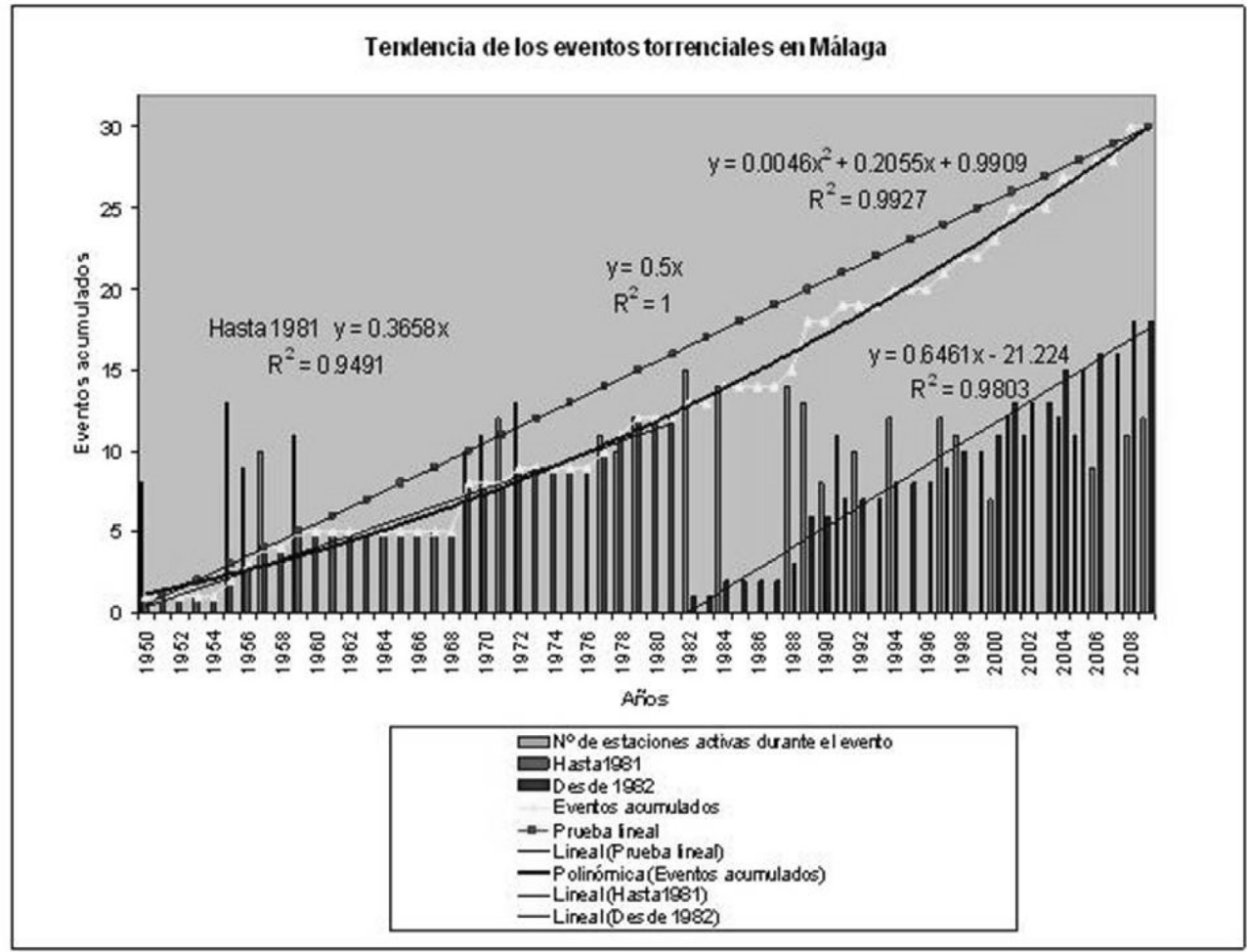

viene a ser similar (entre 8 y 13 estaciones hasta 1979, y entre 8 y 15 hasta 2009), lo que permite que los datos sean comparables. El punto de inflexión en los años 80 coincide con lo planteado por Ruiz Sinoga y Leon Gross (2012) en su análisis de las sequías en el mismo entorno y periodo. Esto puede ser remarcado, de acuerdo con el análisis lineal hasta el año 81 y desde dicho año a la actualidad, con unos coeficientes de determinación muy elevados.

No obstante, el análisis de los volúmenes de precipitación de cada evento (tabla 7 y Figura 3), muestra una cierta tendencia a la disminución, semejante (incluso en su baja fiabilidad estadística) a la tendencia general al descenso de los volúmenes anuales de precipitación en la ciudad de Málaga, estimada por Ruiz Sinoga et al. (2010) en 0'57 mm/año. También es significativa la tendencia a la disminución de los aguaceros torrenciales generalizados a todo el entorno de Málaga. Ambos efectos contribuyen a la disminución del módulo pluviométrico anual en un medio como el mediterráneo, que tanto depende en sus volúmenes finales de las lluvias torrenciales. Tales eventos se produjeron en los años 1955, 1956, 1978, 1982 y 2004. Sin embargo, hubo otros, que por su magnitud fueron importantes, aunque solo se produjeron en una o pocas estaciones en la ciudad de Málaga; es el caso de octubre de 1988 o 14 de noviembre de 1989, en los que se sufrieron inundaciones en el Guadalhorce que, como es el caso del segundo, llegaron a repercutir en la ciudad de Málaga. 
TENDENCIA DE MÁXIMOS VOLÚMENES EN EVENTOS DE > 100 MM/24 H SOBRE MÁLAGA CIUDAD

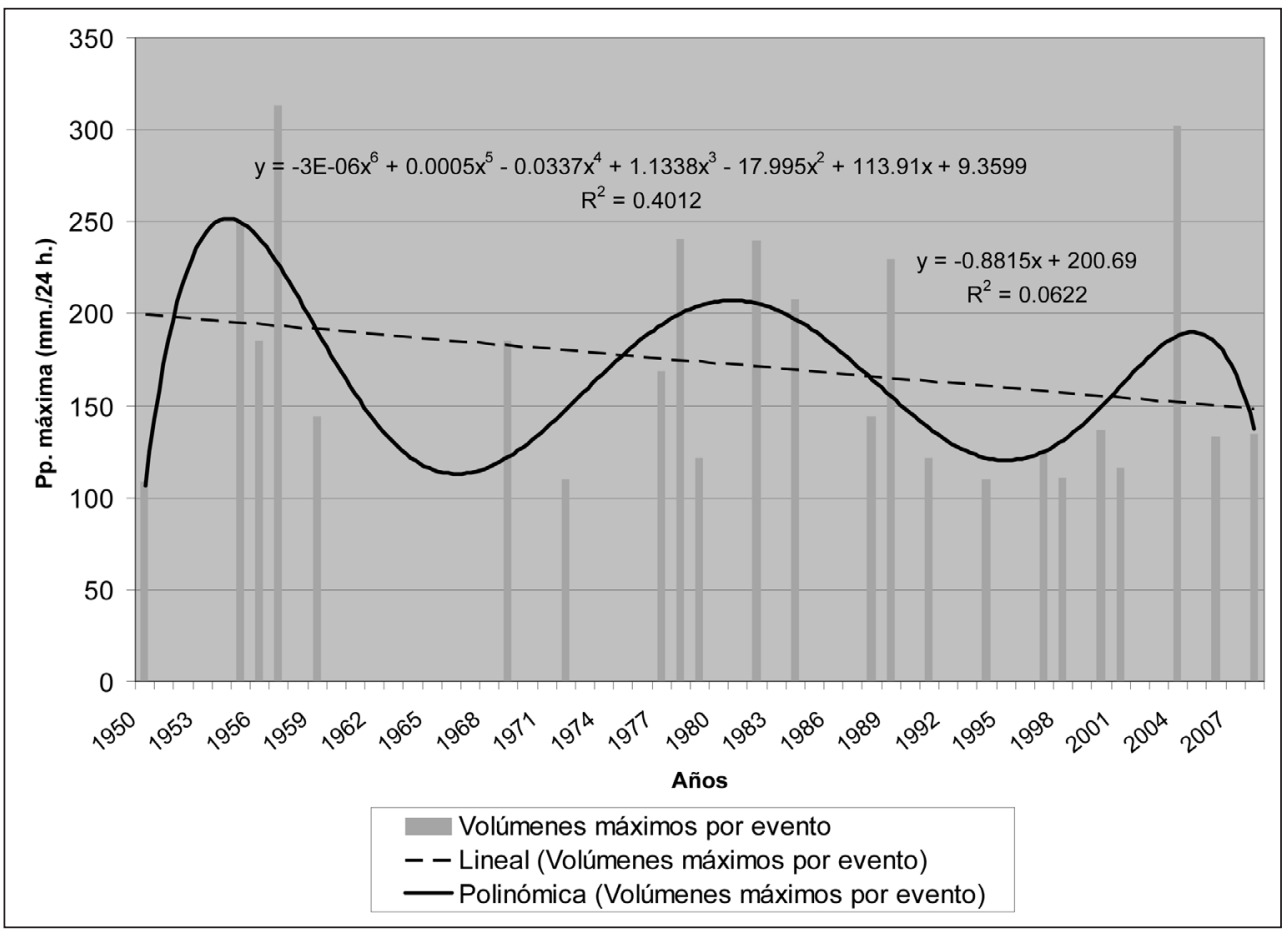

\subsection{Cartografía de la probabilidad de eventos torrenciales}

Para analizar espacialmente estas tendencias, se han realizado varios mapas de probabilidad de afectación de las precipitaciones torrenciales en el entorno de la ciudad de Málaga, para cada una de las estaciones consideradas.

La probabilidad para todo el periodo considerado alcanza los valores máximos en el área norte de la ciudad y (Fig. 4.1), y en general en las áreas más elevadas (Sierra de Mijas, Montes de Málaga), teniendo una mayor frecuencia en la ciudad que en el Valle del Guadalhorce o el área oriental de la ciudad de Málaga. Esto puede ser matizado al considerar la probabilidad de que se presente una precipitación torrencial cuando la situación meteorológica desencadena un aguacero de estas características en cualquier punto del entorno de Málaga (Fig. 4.2), puesto que si bien los resultados son parecidos, esta es más alta en el área del aeropuerto y en la zona a levante de la ciudad. Los resultados de ambos mapas contrastan con la segregación por áreas realizada en el punto 3.1 de este trabajo, donde se señalaba el área de Guadalhorce como aquélla en la que más eventos se recogían; los datos se diluyen al analizarlos individualizadamente.

Un diferente escenario aparece al considerar las precipitaciones torrenciales generadas por DANAsf frente a las que tienen por origen flujos fríos. La comparación de ambos mapas muestra probabilidades cercanas en ambos casos, si bien con matices importantes: los Mon- 
Figura 4.1 y 4.2 .

MAPAS DE \% DE PROBABILIDAD DE EVENTOS > 100 MM/24 H SOBRE MÁLAGA CIUDAD
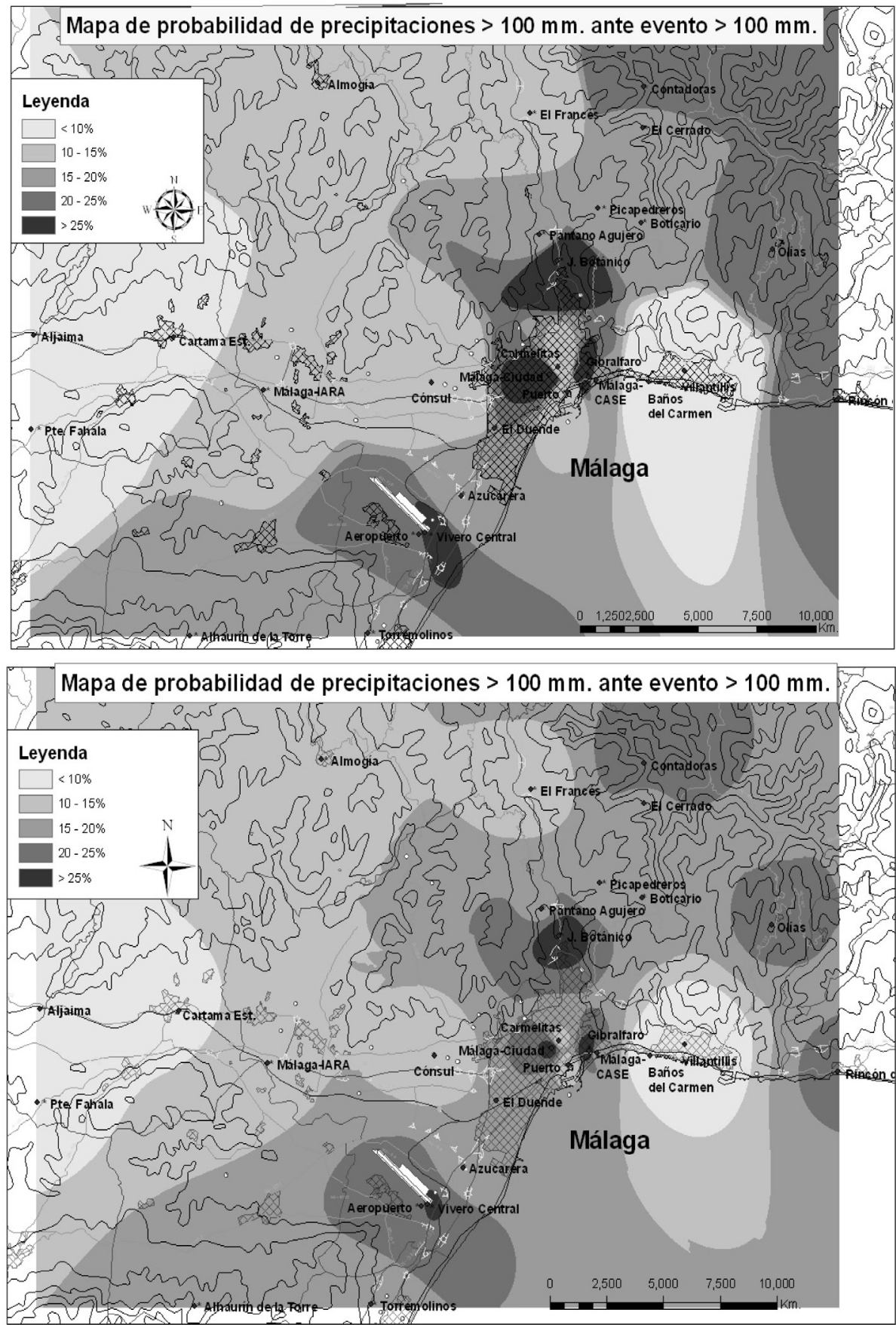
Figura 5.1 y 5.2

MAPA DE \% DE PROBABILIDAD DE > 100 MM ANTE GOTAS Y FLUJOS FRÍOS DE > 100 MM/24 H EN MÁLAGA CIUDAD $\%$ PROBABILITY MAP OF >100 MM. WITH CUT-OFF LOW AND FLOWS OF >100 MM/24H ON MALAGA-CITY
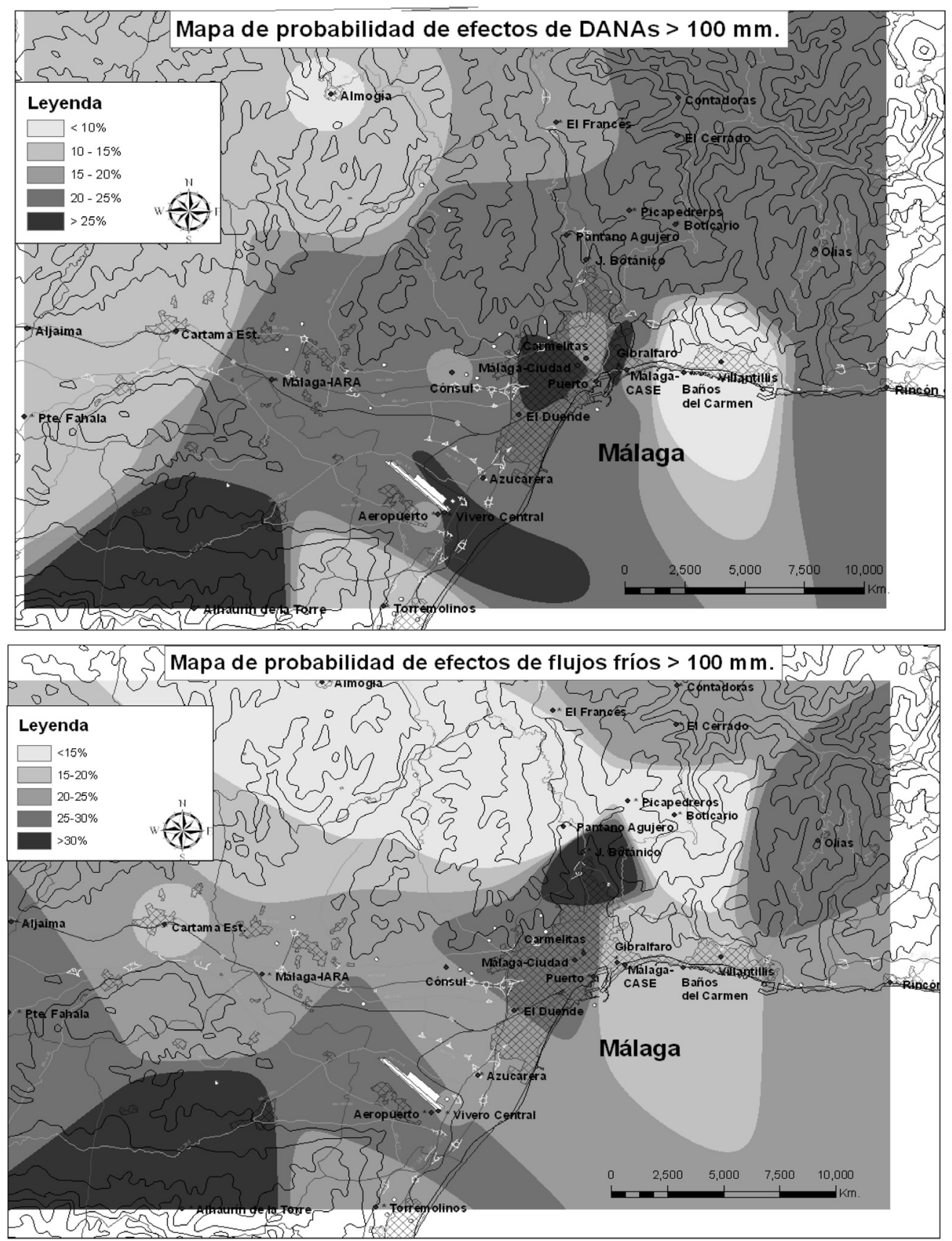
Figura 6.1 y 6.2

MAPA DE \% DE PROBABILIDAD DE > 100 MM/24 H SEGÚN VIENTO DOMINANTE
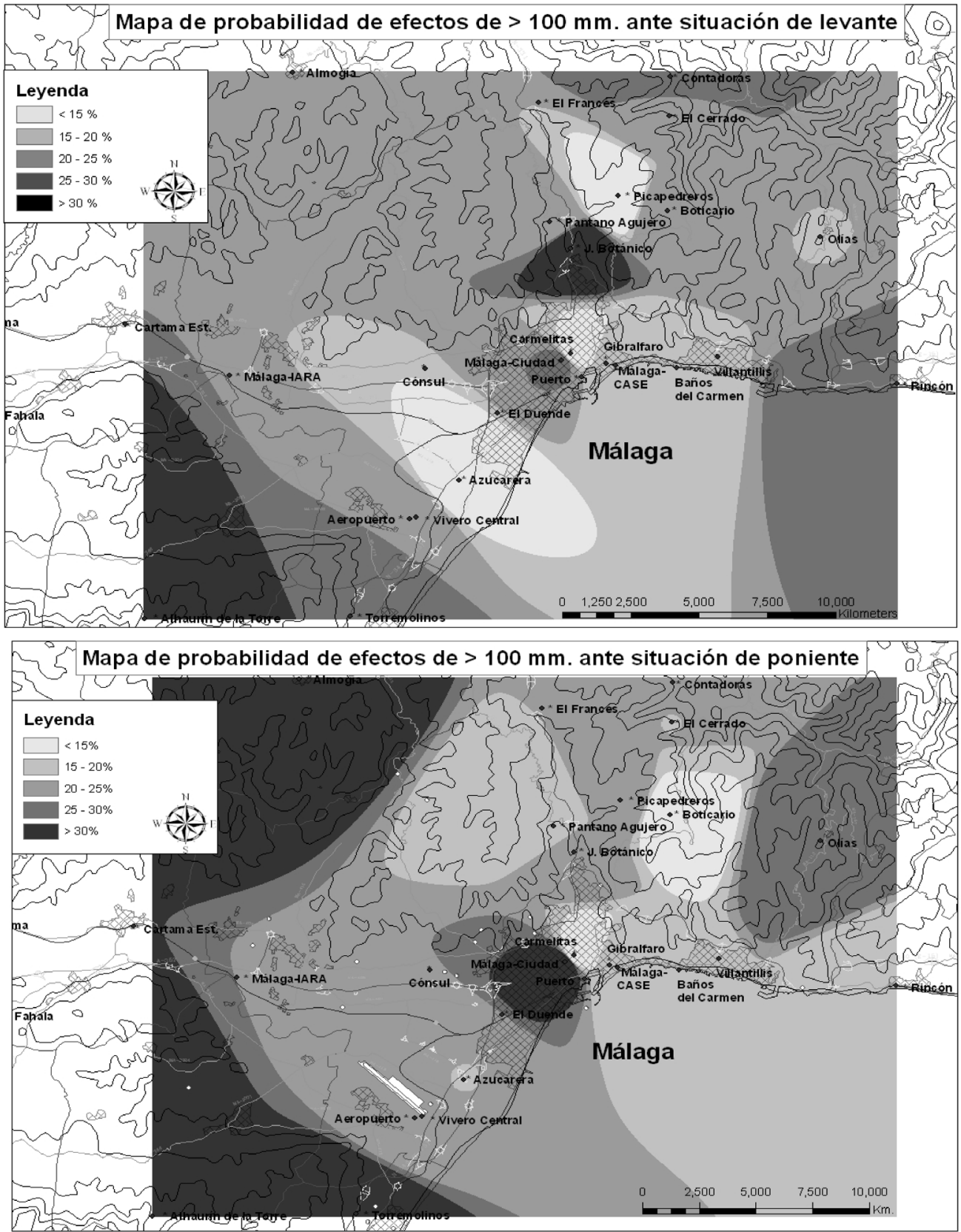
tes de Málaga parecen menos afectados por flujos fríos que por el efecto de DANAs; siendo el centro urbano y área del Guadalhorce al oeste de la ciudad las zonas en las que una mayor importancia adquieren los efectos de las DANAs. En conjunto, tal como mostraban las figuras 5.1 y 5.2, parece darse una mayor probabilidad de efectos tanto de flujos como de DANAs en el área costera.

Por último, se ha analizado la probabilidad de precipitaciones torrenciales en function de los vientos dominantes, (Fig. 6.1 y 6.2) ya sean de levante (agrupado este, sureste y sur) o de viento de poniente (agrupados suroeste y oeste). Los resultados muestran cómo el viento de levante afecta más a las zonas más elevadas de la ciudad, alcanzando sus menores probabilidades en el centro urbano; sin embargo, son las situaciones con viento de poniente las que presentan mayor probabilidad tanto en la ciudad como en el valle del Guadalhorce, con mínimos en algunas áreas de los Montes de Málaga y área oriental de la ciudad. Conviene recordar que el tipo de viento que con más frecuencia genera precipitaciones en la ciudad de Málaga es el de componente suroeste y no el levante como sucede hacia el Mediterráneo (datos de AEMET desde 1942), a la vez que los vientos más frecuentes en el Aeropuerto de Málaga son los del tercer y cuarto cuadrante, que suponen una frecuencia casi doble (63\%) que los del segundo y tercero (33\%).

\section{DISCUSIÓN}

Los eventos torrenciales son aleatorios en el tiempo y en el espacio, lo que puede obedecer a causas topográficas cuyo estudio no puede omitir cualquier otro dato de carácter puntual, aunque en las series estadísticas quede invalidado para conocer el módulo pluviométrico o, incluso, el valor medio de las precipitaciones extremas. De hecho, en mediciones de parcelas experimentales en las que utilizan pluviógrafos instalados para el estudio concreto de la parcela, las series carecen de validez estadística en la inmensa mayoría de los casos... y sin embargo están midiendo un hecho significativo: la intensidad de las precipitaciones.

Si para el estudio de eventos torrenciales, se analiza una densa (aunque variable temporal y numéricamente) red de datos, no debe ser descartada aunque su validez estadística sea discutible. El criterio de presencia-ausencia es fundamental en estos casos. Si un evento torrencial se registra simultáneamente en numerosas estaciones (independientemente de la validez de éstas) su repercusión será más importante que si el mismo se presenta aislado, con escasa repercusión en las estaciones periféricas.

La variabilidad espacio-temporal de las precipitaciones torrenciales incluso en areas reducidas determina la posibilidad de que tales eventos sean más frecuentes de lo que los observatorios nos indican. En un área como la ciudad de Málaga (32 km² aproximadamente), el número de estaciones pluviométricas de series estadísticamente fiables y continuas a lo largo de más de 30 años es escaso, y sin embargo, es habitual que algunos eventos torrenciales hayan sido registrados en unas sí y en otras no, o bien (lo más frecuente) no hayan alcanzado intensidad significativa. Y sin embargo, tanto unos como otros han sido eventos de importantes repercusiones para la gestión urbana.

Además de la localización preferente, es interesante conocer las tendencias temporales de las precipitaciones, y así en los datos aquí analizados se revela una cierta tendencia al incremento de la frecuencia a la vez que un cierto decrecimiento en el volumen. Ambos ele- 
mentos pueden ser de interés para la gestión urbana (obras de colectores urbanos, limpiezas de cauces con mayor frecuencia).

Con estas premisas, conocer la situación atmosférica que determina los criterios de ausencia-presencia en relación con la topografía puede ser una línea que pretendemos establecer en este estudio, siendo obviamente necesario ahondar en el papel preciso de los factores altitud-exposición.

\section{CONCLUSIONES}

La frecuencia de aguaceros torrenciales es muy superior a la que los análisis de una sola estación llegan a mostrar o prever mediante el análisis probabilístico. A lo largo de 59 años se han producido 30 aguaceros de esta naturaleza en algún punto de la ciudad de Málaga o periférico a la misma, lo que implicaría una recurrencia conjunta aproximada de un evento cada 2 años. El análisis de la serie revela cómo esta frecuencia tiende a acelerarse en los últimos años, aunque el volumen máximo tienda a ser ligeramente inferior. Esta sería una de las claves del descenso del módulo pluviométrico anual, si consideramos que buena parte del volumen anual de precipitación en el Mediterráneo depende de las cantidades precipitadas en eventos de gran intensidad.

Atendiendo a su distribución espacial, se muestra cómo el área urbana de Málaga es especialmente sensible ante estos eventos, con una alta probabilidad que alcanza sus mayores valores ante situaciones de gotas frías y flujos de poniente.

Del análisis de los eventos generalizados que han provocado inundaciones en la ciudad de Málaga o su entorno se desprende que existen tres situaciones estándar que suelen dar lugar a ello:

1.- Depresión fría (DANA + sistema depresionario en superficie) sobre África (y anticiclón en las islas Británicas), que introduce viento de levante. Entre los aguaceros de especial intensidad, ese el modelo que más comúnmente se generaliza en los observatorios y que, por ello, suele producir los mayores estragos sobre la ciudad de Málaga.

2.- Depresión fría sobre el Golfo de Cádiz-San Vicente, aunque, de modo secundario, si la depresión es muy profunda, también pueden afectar las situadas frente a Galicia. En estos casos de borrasca atlántica puede darse viento de poniente (que es el responsable de la lluvia frecuente o de baja intensidad en la ciudad de Málaga), aunque la evolución de la depresión hacia la ciudad de Málaga suele dar lugar a que el viento role a levante a lo largo del evento.

3.- El flujo en altura sin embolsamiento definido suele provocar con menor frecuencia eventos generalizados sobre la ciudad de Málaga, pero suele ser responsable de aguaceros más o menos aislados de gran intensidad.

Sin embargo, el análisis de los efectos sobre el entorno de la ciudad de Málaga revela que no suelen afectar por igual las situaciones de levante que las de poniente. El centro urbano es más sensible a situaciones de poniente, en tanto que las zonas elevadas (Montes de Málaga, Sierra de Mijas) son más sensibles al levante.

Las DANAs afectan a una mayor proporción de la zona urbana que los eventos torrenciales con flujo frío ( más cercana a la costa y al área oriental de los Montes de Málaga es más proclive a eventos torrenciales; eventos que, cuando se analiza su recurrencia aisladamente (probabilidad de 
que superen $100 \mathrm{~mm}$ ante un evento que supere los $100 \mathrm{~mm}$ en cualquier lugar de la ciudad) se verifican, dando especial peso a la zona del aeropuerto, norte de la ciudad y área oriental de los Montes de Málaga.

Este hecho es de suma importancia para la gestión urbana en la que no importa en qué punto de la ciudad suceda, sino, simplemente que suceda. Considerando que la red de pluviómetros urbanos no es ni lo densa ni todo lo continua que sería deseable para una ciudad de más de $30 \mathrm{~km}^{2}$ de superficie (>60 si incluimos área periurbana), es muy posible que la probabilidad real de precipitación intensa en algún punto de la ciudad sea superior a lo calculado a partir de las estaciones disponibles. Atender a la situación sinóptica puede servir para conocer, en función de la cartografía presentada, cuáles son las áreas de mayor probabilidad de lluvia torrencial.

\section{REFERENCIAS}

ABC: http://hemeroteca.abc.es/nav/Navigate.exe/hemeroteca/madrid/abc/1955/11/02/005.html.

ALBENTOSA SÁNCHEZ, L. (1991): El clima y las aguas. Col. Geografía de España. Ed. Síntesis. Madrid. 240 pp.

CAMARASA BELMONTE, A.M., SORIANO GARCÍA, J. y LÓPEZ-GARCÍA, M.J. (2010): «The effect of observation timescales on the characterisation of extreme Mediterranean precipitation». Advances in Geoscience, 26, 61-64, 2010 doi:10.5194/adgeo26-61.

CAPEL MOLINA, J.J. (1990): «Ciclogénesis violenta en el Mediterráneo. La inundación de Málaga de Noviembre de 1989». Papeles de Geografía, nº 16: 9-33.

CAPEL MOLINA, J.J. (2000): El clima de la Península Ibérica. Ariel Geografía. Barcelona, $281 \mathrm{pp}$.

DE LUIS, M., GONZÁLEZ-HIDALGO, J. C., RAVENTÓS, J., SÁNCHEZ, J. R., y CORTINA, J. (1997): «Distribución espacial de la concentración y agresividad de la lluvia en el territorio de la comunidad Valenciana». Cuaternario y Geomorfologia, 11 (3-4), 33-44.

DIARIO SUR: http://servicios .diariosur.es/fijas/esp/malagasigloXX.

ELÍAS CASTILLO, F. y RUIZ BELTRÁN, F. (1979): Precipitaciones máximas en España. Ministerio Agricultura. Madrid. 545 pp.

EL PAÍS: http://www.elpais/articulo/espana/MALAGA

FONT TULLOT, I. (1983): Climatología de España y Portugal. Instituto Nacional de Meteorología, Madrid.

LANA, X., BURGUEÑO, A., MARTÍNEZ, M.D., y SERRA, C. (2009): «Una revisión de los análisis estadísticos de las precipitaciones diarias y mensuales en Cataluña». Tethys, 6, 15-30.

LLASAT, M.C., BARRIENDOS, M., BARRERA, A., RIGO, T. (2005): «Floods in Catalonia (NE Spain) since the 14th century. Climatological and meteorological aspects from historical documentary sources and old instrumental records». Journal of Hydrology, 313: 32-47.

LÓPEZ GÓMEZ, A. (1969): «El supuesto monzón de la Península Ibérica». En Aportación española al XXI Congreso Geográfico Internacional. Instituto Geográfico y Catastral. Madrid.pp. 72-88.

LÓPEZ GÓMEZ, A. (1983): «Las lluvias catastróficas mediterráneas». Estudios Geográfi$\cos , \mathrm{n}^{\circ}$ 44: 170/171: 11-29. 
MARTÍN LEÓN, F. (2003): Las gotas frías / DANAS. Ideas y conceptos básicos. Servicio de Técnicas de Análisis y Predicción. Instituto Nacional de Meteorología, 14 pp.

MARTÍN VIDE, J. (2004): «Spatial distribution of a daily precipitation concentration index in Peninsular Spain». International Journal of Climatology. 24, 959-971.

MARTÍN VIDE, J., SÁNCHEZ-LORENZO, A., LÓPEZ BUSTINS, J. A., CORDOBILLA, M. J., GARCIA MANUEL, A., and RASO, J. M. (2008): «Torrential rainfall in northeast of the Iberian Peninsula: synoptic patterns and WeMO influence». Advances in Science and Research, 2, 99-105.

MARTÍN, M.L., SANTOS-MUÑOZ, D., MORATA, A, LUNA, M.Y., VALERO, F. (2006): «An objectively selected case study of a heavy rain event in the Mediterranean Basin: A diagnosis using numerical simulation». Atmospheric Research, 81: 187-205. Elsevier.

NEPPEL, L., ARNAUD, P., LAVABRE, J. (2007): «Connaissance régionale des pluies extrêmes. Comparaison de deux approches appliquées en milieu méditerranéen». C.R. Geosciences, 339: 820-830. Elsevier.

PASCUAL AGUILAR, J.A., AÑO VIDAL, C., SÁNCHEZ DÍAZ, J., MASIÁ MIRA, F. J., y ARNAU ROSALÉN, E. (2001): «Valoración de la idoneidad de los índices PCI y MFI para estimar la concentración y agresividad de las precipitaciones en la Comunidad Valenciana». Cuaternario y Geomorfología, 15 (3-4), 77-88.

PONS MARTI, V., y SORIANO SOTO, M. D. (1994): «Valores de torrencialidad R. Su cálculo y distribución en la provincia de Alicante». Cuadernos de Geografía, 56. 163-174.

RODRIGO, F. S. y BARRIENDOS, M. (2008): «Reconstruction of seasonal and annual rainfall variability in the Iberian peninsula (16th-20th centuries) from documentary data». Global and Planetary change, 63: 243-257.

RUIZ SINOGA, J.D., GARCÍA MARÍN, R., MARTÍNEZ MURILLO, J.F., GABARRÓN GALEOTE, M.A. (2010): «Precipitation dynamics in southern Spain: trends and cycles». Int. J. Climatol. DOI: 10.1002/joc.2235.

SENCIALES GONZÁLEZ, J.M. (1995): La cuenca del Río Vélez. Estudio HidrográficoUniversidad de Málaga. Tesis Doctoral. Servicio de Publicaciones de la Universidad de Málaga. Ed. Microfichas.

SENCIALES GONZÁLEZ, J.M. (1997): La intensidad de las precipitaciones en la ciudad de Málaga. Baetica, 19: 301-312. Número Extraordinario «Homenaje al Profesor García Manrique».

TURCO, M. y LLASAT, M.C. (2011): T»rends in indices of daily precipitation extremes in Catalonia (NE Spain), 1951-2003». Natural Hazards and Earth System Sciences, 11, 3213-3226

WETTERZENTRALE (2010): http://www.wetterzentrale.de/topkarten/fsraeur/html

ZIPSER, E.J. (1982): «Use of a conceptual model of the life-cycle of mesoscales convective systems to improve very-short range forecast», en Browning, K. (ed.): Nowcasting. Academic Press, New York, 191-204. 